\title{
Associação entre os fatores de risco para os transtornos mentais comuns em estudantes de fisioterapia
}

\author{
Association between risk factors for common mental \\ disorders in physical therapy students
}

\section{Asociación entre factores de riesgo de trastornos mentales comunes en estudiantes de fisioterapia}

\author{
Biatriz Araújo Cardoso Dias ${ }^{1}$, Aline Silva Castro ${ }^{1}$, Cristiane de Fátima \\ Pimenta da Costa ${ }^{2}$, Jamylle Silva Campos ${ }^{1}$, Leonardo Breno do \\ Nascimento de Aviz ${ }^{1}$, Náthila Lorrana Silva Cardoso ${ }^{2}$, Shaumin \\ Vasconcelos $\mathrm{Wu}^{1}$, Norma Sueli dos Reis Cardoso ${ }^{3}$, Angélica \\ Homobono Nobre ${ }^{1}$, George Alberto da Silva Dias ${ }^{1}$
}

1.Fisioterapeuta. Departamento de Ciências do Movimento Humano. Universidade do Estado do Pará (UEPA), Belém-PA, Brasil.

2.Graduação em Fisioterapia. Departamento de Ciências do Movimento Humano. Universidade do Estado do Pará (UEPA), Belém-PA, Brasil.

3. Pedagoga. Departamento de Ciências do Movimento Humano. Universidade do Estado do Pará (UEPA), Belém-PA, Brasil.

\begin{abstract}
Resumo
Objetivo. Relacionar os fatores de risco para os transtornos mentais comuns em estudantes de diferentes períodos do curso de Fisioterapia pertencentes ao ensino por metodologia ativa e tradicional. Método. Trata-se de um estudo observacional, descritivo e analítico, do tipo transversal, no qual participaram 123 discentes do curso de Fisioterapia, com aplicação de questionário sociodemográfico e o Self-Reporting Questionnaire (SRQ-20). Resultado. Participaram do estudo 72 alunos inseridos na metodologia ativa (MA) e 51 ainda pertencentes a metodologia tradicional (MT). A maioria dos alunos são de etnia parda, solteiros e com renda familiar de 2 a 6 salários, realizam atividade extracurricular, e um número considerável de alunos não realizam nenhuma atividade de lazer e nem pratica atividade física. Foi possível observar que houve grande número de casos suspeitos de transtornos mentais $(p=0,005)$. Em ambos os sexos foi possível notar que aqueles alunos que não praticam atividade física aumentam em 2,85 vezes a chance de transtorno mental. Conclusão. Pode-se concluir que os alunos que não praticam atividade física aumentam em 2,85 vezes a chance de desenvolver transtorno mental. E quando estratificado por sexo e metodologia de ensino, as alunas pertencentes à metodologia ativa aumentam em 4,8 vezes a chance de desenvolver transtorno mental.
\end{abstract}

Unitermos. Transtornos Mentais; Saúde mental; Estudantes

\footnotetext{
Abstract

Objectives. Relate the risk factors for common mental disorders in students from different periods of the Physiotherapy course belonging to teaching by active and traditional methodology. Method. This is an observational, descriptive, and analytical cross-sectional study, in which 123 students of the Physiotherapy course participated, with the application of a sociodemographic questionnaire and the Self-Reporting Questionnaire (SRQ-20). Result. Seventy-two students enrolled in the active methodology (AM) and 51 still belonging to the traditional methodology (TM) participated in the study. Most students are brown, single and with a family income of 2 to 6 salaries, perform extracurricular activities, and a considerable number of students do not perform any leisure activity or practice physical activity. It was possible to observe that there were many suspected cases of mental disorders $(p=0.005)$. In
} 
both sexes it was possible to notice that those students who do not practice physical activity increase the chance of mental disorder by 2.85 times. Conclusion. It can be concluded that students who do not practice physical activity increase 2.85 times the chance of developing mental disorder. And when stratified by gender and teaching methodology, students belonging to the active methodology increase by 4.8 times the chance of developing mental disorder.

Keywords. Mental Disorders; Mental health; students

\section{Resumen}

Objetivo. Relacionar los factores de riesgo de trastornos mentales comunes en estudiantes de diferentes períodos del curso de Fisioterapia pertenecientes a la enseñanza por metodología activa y tradicional. Método. Se trata de un estudio transversal observacional, descriptivo y analítico, en el que participaron 123 alumnos del curso de Fisioterapia, con la aplicación de un cuestionario sociodemográfico y el Self-Reporting Questionnaire (SRQ-20). Resultado. Setenta y dos estudiantes inscritos en la metodología activa (MA) y 51 que todavía pertenecen a la metodología tradicional (MT) participaron en el estudio. La mayoría de los estudiantes son marrones, solteros y con un ingreso familiar de 2 a 6 salarios, realizan actividades extracurriculares, y un número considerable de estudiantes no realizan ninguna actividad de ocio o practican actividad física. Era posible observar que había un gran número de casos sospechosos de trastornos mentales $(p=0,005)$. En ambos sexos fue posible notar que aquellos estudiantes que no practican la actividad física aumentan la probabilidad de trastorno mental en 2,85 veces. Conclusión. Se puede concluir que los estudiantes que no practican la actividad física aumentan 2,85 veces, la probabilidad de desarrollar trastorno mental. Y cuando están estratificados por el género y la metodología de enseñanza, los estudiantes pertenecientes a la metodología activa aumentan en 4,8 veces la probabilidad de desarrollar trastorno mental.

Palabras clave. Trastornos mentales; Salud mental; estudiantes

Trabalho realizado na Universidade do Estado do Pará (UEPA), Belém-PA, Brasil.

Conflito de interesse: não

Recebido em: 06/04/2021

Aceito em: 08/09/2021

Endereço para correspondência: George Alberto da Silva Dias. Universidade do Estado do Pará (UEPA). Rua do Una 156. Telégrafo. CEP 66050-540. Belém-PA, Brasil. Telefone: (91) 3131-1708. E-mail: george@uepa.br

\section{INTRODUÇÃO}

A temática relacionada à saúde mental, tornou-se algo relevante em diversos âmbitos. Em grande parte, pelas implicações que esta suscita quando não evidenciada de forma adequada, reverberando em quadros de ansiedade, depressão, transtornos de humor, entre outros. Sendo inegável que suas problemáticas implicam em efeitos tanto na qualidade de vida, quanto nas atividades laborais e nas relações sociais ${ }^{1}$. Sabe-se também que os transtornos mentais provocam inúmeras mudanças biopsicossociais, porém mesmo com tal fato a procura por ajuda profissional ainda é baixa, em razão das barreiras e impedimentos que 
cercam esse tema e que consequentemente postergam o tratamento adequado. Logo, a prevenção e promoção da saúde mental acabam se tornando essenciais ${ }^{2}$.

O ambiente universitário representa uma série de mudanças na vida do indivíduo, onde se tem uma grande carga de responsabilidades, com cobranças internas e externas; pessoais, familiares e comunitárias. Nesse cenário, estão inclusos fatores de risco de condições como o estresse, ansiedade e depressão. Dessa forma, fica evidente a necessidade de atenção à saúde como um todo ao grupo universitário, buscando evitar um número alarmante de pessoas comprometidas, não só no que diz respeito à saúde mental, mas as suas consequências no âmbito pessoal e social, envolvendo o aproveitamento da graduação e consequentemente a expectativa profissional3.

Tendo por base a relação meio acadêmico e saúde mental é possível encontrar pesquisas afirmando que os cursos da área de saúde são os que mais afetam psicossocialmente os alunos, havendo nestes, inclusive alguns fatores determinantes para geração de distúrbios psíquicos. Tais fatores estão relacionados a percepção quanto ao curso, condições de saúde, adaptação, inserção social e fatores socioeconômicos ${ }^{4}$.

Observando-se o curso de Fisioterapia especificamente, é possível notar que alguns autores ${ }^{5}$ revelam que 0 rendimento e a evasão acadêmica estão vinculados ao nível de saúde mental. Além disso, fatores como baixa idade e períodos iniciais e finais do curso são vistos recorrentemente 
associados a possíveis distúrbios, uma vez que indivíduos muito jovens ainda não tem maturidade suficiente para lidar com as exigências do ensino superior, e aliado a isso os períodos iniciais do curso que é um período de adaptação podem gerar distúrbios psíquicos, assim como períodos finais geram maior pressão por ser uma fase de transição para a vida profissional ${ }^{5}$.

Desse modo há evidências em pesquisas e estudos científicos que as faculdades e universidades são locais propícios para o desenvolvimento de transtornos mentais ${ }^{3-5}$. Sendo assim, este estudo objetiva relacionar os fatores de risco para os transtornos mentais comuns em estudantes do curso de Fisioterapia.

\section{MÉTODO}

\section{Aspectos éticos}

A pesquisa teve início após aprovação do Comitê de Ética em Pesquisa da Universidade do Estado do Pará (UEPA) (Parecer no 2.710.595; CAAE: 88961118.6.0000.8130). Trata-se de um estudo observacional, descritivo e analítico, com método de abordagem quantitativa, seguindo as normas da Resolução 466/12 do Conselho Nacional de Saúde, relativa à pesquisa com seres humanos. Todos os participantes assinaram o Termo de Consentimento Livre e Esclarecido e/ou o Termo de Assentimento Livre e Esclarecido. 


\section{Amostra}

Participaram do estudo 123 discentes do curso de Fisioterapia da UEPA regularmente matriculados, sendo selecionados por meio da amostragem não probabilística por conveniência e foram avaliados no período de agosto a dezembro de 2018.

Como critérios de inclusão optou-se por discentes de ambos os sexos, maiores e menores de 18 anos do curso de Fisioterapia da UEPA e que quisessem participar da pesquisa. Foram excluídos aqueles que não assinaram formalmente a sua participação no estudo e que se recusaram a responder a um dos questionários propostos.

\section{Procedimentos}

Os instrumentos de coleta de dados utilizados na pesquisa foram autoaplicáveis facilitando a fidedignidade nas respostas. No primeiro momento os participantes encontravam-se dentro do ambiente universitário onde foram abordados e explicado o estudo. No segundo momento, após o consentimento dos participantes, distribuiu-se os questionários aos alunos para preenchimento das informações podendo ser entregue no dia seguinte após seu consentimento.

Foi utilizado um questionário sociodemográfico contando informações sobre sexo, etnia, estado civil, renda familiar, reprovação no curso, atividade extracurricular, lazer (apresenta atividade prazerosa durante um determinado 
tempo do seu dia) e atividade física (praticar pelo menos duas vezes por semana).

Para o rastreamento de transtornos mentais comuns (TMC), utilizou-se o Self-Reporting Questionnaire (SRQ-20). Os TMC representam as patologias mais comuns, porém menos graves dos transtornos mentais, incluindo sintomas como alterações na memória, insônia, irritabilidade, fadiga, tremores, queixas gástricas, cefaleia, falta de apetite e dificuldade de concentração e na tomada de decisões. Este instrumento é composto por 20 perguntas com respostas "sim" ou "não", sendo 4 perguntas sobre sintomas físicos e 16 sobre desordens psicoemocionais. Os sintomas avaliados foram referentes aos últimos 30 dias, e a cada resposta "sim" foi atribuído um ponto, resultando em uma pontuação final que variou de 0 a 20 pontos, onde 0 significa "nenhuma probabilidade" a 20 que é "extrema probabilidade". Os alunos foram distribuídos em dois grupos, de acordo com a pontuação no SRQ-20, pois o ponto de corte é diferente. Homens com pontuação menor ou igual a cinco e mulheres com pontuação menor ou igual a sete foram classificados como "não suspeitos". Homens com pontuação maior ou igual a seis e mulheres com pontuação maior ou igual a oito foram classificados como "suspeitos"6,7.

\section{Análise estatística}

Adotou-se o software Excel $\circledast 2010$ para entrada dos dados e confecção das tabelas, bem como o BioEstat 5.0 na análise estatística. As variáveis categóricas foram 
apresentadas como frequências e as numéricas por meio de medidas de tendência central e dispersão. Utilizou-se os testes análise de variância (ANOVA dois critérios), o teste do Qui-quadrado, e na investigação mais detalhada dos fatores de risco comportamentais possivelmente associados ao transtorno mental comum, realizou-se análise geral e estratificada por sexo e metodologia (ativa e tradicional), sendo então empregado o cálculo das razões de chances de prevalência (ORp) com intervalo de confiança (IC) de 95\%. Adotou-se o nível alfa de significância de 5\% ( $p \leq 0,05)$.

\section{RESULTADOS}

Participaram do estudo 72 alunos inseridos na metodologia ativa (MA) e 51 pertencentes a metodologia tradicional (MT), totalizando 123 discentes, sendo 33 do

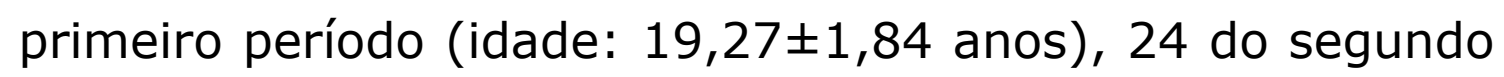
(Idade: $19,41 \pm 1,66$ anos), 15 do terceiro (Idade: $19,87 \pm 0,61$ anos), 26 do quarto (Idade: 22,69 $\pm 2,46$ anos)

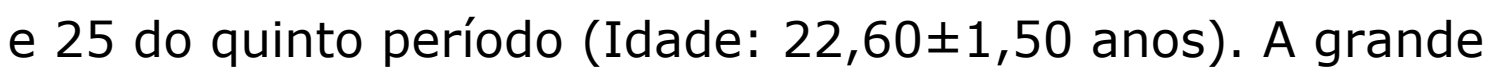
maioria dos alunos são de etnia parda, solteiros e com renda familiar de 2 a 6 salários (Tabela 1). Observou-se ainda que em todos os períodos, algum aluno reprovou em alguma disciplina, a grande maioria realiza atividade extracurricular, e um número considerável de aluno não realiza nenhuma atividade de lazer e nem pratica atividade física (Tabela 1). 
Tabela 1. Perfil sociodemográfico dos alunos do curso de Fisioterapia da UEPA, Belém, Pará, Brasil.

\begin{tabular}{|c|c|c|c|c|c|}
\hline \multirow{2}{*}{ VARIÁVEIS } & \multicolumn{3}{|c|}{ MA } & \multicolumn{2}{|c|}{ MT } \\
\hline & $1^{\circ}$ período & $2^{\circ}$ período & $3^{\circ}$ período & $4^{\circ}$ período & $5^{0}$ período \\
\hline \multicolumn{6}{|l|}{ Sexo } \\
\hline Masculino & $11(33,3)$ & $5(20,8)$ & $7(46,7)$ & $7(26,9)$ & $5(20,0)$ \\
\hline Feminino & $22(66,7)$ & $19(79,2)$ & $8(53,3)$ & $19(73,1)$ & $20(80,0)$ \\
\hline \multicolumn{6}{|l|}{ Etnia } \\
\hline Branco & $5(15,2)$ & $2(8,3)$ & $4(25,0)$ & $4(15,4)$ & $6(24,0)$ \\
\hline Pardo & $9(27,3)$ & $14(58,3)$ & $9(56,3)$ & $13(50,0)$ & $11(44,0)$ \\
\hline Negro & $3(9,1)$ & $4(16,7)$ & $2(12,5)$ & $1(3,8)$ & $5(20,0)$ \\
\hline Não declarou & $16(48,5)$ & $4(16,7)$ & $1(6,2)$ & $8(30,8)$ & $6(24,0)$ \\
\hline \multicolumn{6}{|l|}{ Estado civil } \\
\hline Solteiro & $32(97,0)$ & $24(100,0)$ & $16(100,0)$ & $24(92,3)$ & $\begin{array}{c}25 \\
(100,0)\end{array}$ \\
\hline Casado & $1(3,0)$ & - & - & $2(7,7)$ & - \\
\hline \multicolumn{6}{|l|}{ Renda familiar } \\
\hline Não declarou & $1(3,0)$ & - & - & - & - \\
\hline < 1 salário & $2(6,1)$ & - & $1(6,7)$ & $1(3,8)$ & - \\
\hline 1 salário & $3(9,1)$ & $4(16,7)$ & $1(6,7)$ & $2(7,7)$ & $2(8,0)$ \\
\hline 2 a 3 salários & $14(42,4)$ & $12(50,0)$ & $5(33,3)$ & $15(57,7)$ & $10(40,0)$ \\
\hline 5 a 6 salários & $9(27,3)$ & $6(25,0)$ & $6(40,0)$ & $5(19,2)$ & $7(28,0)$ \\
\hline$>7$ salários & $3(9,1)$ & $2(8,3)$ & $2(13,3)$ & $3(11,5)$ & $6(24,0)$ \\
\hline \multicolumn{6}{|l|}{ Reprovou } \\
\hline Sim & $1(3,0)$ & $3(12,5)$ & $4(25,0)$ & $6(23,1)$ & $5(20,0)$ \\
\hline Não & $32(97,0)$ & $21(87,5)$ & $12(75,0)$ & $20(76,9)$ & $20(80,0)$ \\
\hline \multicolumn{6}{|l|}{$\begin{array}{l}\text { Atividade } \\
\text { extracurricular }\end{array}$} \\
\hline Sim & $30(90,9)$ & $21(87,5)$ & $14(87,5)$ & $23(92,0)$ & $21(84,00$ \\
\hline Não & $3(9,1)$ & $3(12,5)$ & $2(12,5)$ & $2(8,0)$ & $4(16,0)$ \\
\hline \multicolumn{6}{|l|}{ Lazer } \\
\hline Sim & $23(69,7)$ & $16(66,7)$ & $12(75,0)$ & $15(57,7)$ & $14(56,0)$ \\
\hline Não & $10(30,3)$ & $8(33,3)$ & $4(25,0)$ & $11(42,3)$ & $11(44,0)$ \\
\hline \multicolumn{6}{|l|}{ Atividade física } \\
\hline Sim & $19(57,6)$ & $10(41,7)$ & $9(56,3)$ & $12(48,0)$ & $14(56,0)$ \\
\hline Não & $14(42,4)$ & $14(58,3)$ & $7(43,8)$ & $13(52,0)$ & $11(44,0)$ \\
\hline
\end{tabular}

Na análise dos casos suspeitos e não suspeitos para os transtornos mentais comuns entre os alunos do curso de 
Fisioterapia, foi possível observar que houve grande número de casos suspeitos $(p=0,005)$. Porém, não houve diferença entre os casos suspeitos entre os períodos do curso $(p=0,26$; Tabela 2).

Tabela 2. Prevalência dos casos suspeitos e não suspeitos para o transtorno mental comum dos alunos do curso de Fisioterapia da UEPA.

\begin{tabular}{|c|c|c|c|}
\hline \multirow{2}{*}{ Séries do curso } & \multicolumn{2}{|c|}{$\begin{array}{l}\text { SRQ-20 } \\
\text { Alunos do curso de Fisioterapia }(n=123)\end{array}$} & \multirow{2}{*}{ p-valor ${ }^{2}$} \\
\hline & Suspeitos & Não suspeitos & \\
\hline $1^{\circ}$ período & $21(63,6)$ & $12(36,4)$ & \\
\hline $2^{\circ}$ período & $19(79,2)$ & $5(20,8)$ & \\
\hline 30 período & $13(86,7)$ & $2(13,3)$ & 0,26 \\
\hline $4^{\circ}$ período & $23(88,5)$ & $3(11,5)$ & \\
\hline 50 período & $17(68,0)$ & $8(32,0)$ & \\
\hline p-valor ${ }^{1}$ & \multicolumn{2}{|c|}{0,005} & \\
\hline
\end{tabular}

Quando analisado o SQR-20 estratificado por sexo, foi observado que os alunos foram classificados como suspeitos de transtornos mentais comuns em ambos os sexos $(p=0,04)$. O dado mais alarmante foi entre os alunos do terceiro período e as alunas do quarto período, onde todos foram classificados como suspeitos de transtornos mentais. Porém, ao analisar a diferença entre os períodos não houve diferença significante para ambos os sexos (Tabela 3 ). 
Tabela 3. Análise do SQR-20 dos alunos do curso de Fisioterapia da UEPA, Belém, Pará, Brasil.

\begin{tabular}{|c|c|c|c|c|c|c|}
\hline \multirow{2}{*}{$\begin{array}{l}\text { Séries do } \\
\text { curso }\end{array}$} & \multicolumn{2}{|c|}{$\begin{array}{c}\text { SRQ-20 } \\
\text { Homens }(n=35)\end{array}$} & \multirow{2}{*}{$\begin{array}{c}\text { p- } \\
\text { valor }\end{array}$} & \multicolumn{2}{|c|}{$\begin{array}{c}\text { SRQ-20 } \\
\text { Mulheres }(n=88)\end{array}$} & \multirow{2}{*}{$\begin{array}{c}\text { p- } \\
\text { valor }\end{array}$} \\
\hline & Suspeitos & $\begin{array}{c}\text { Não } \\
\text { suspeitos }\end{array}$ & & Suspeitos & $\begin{array}{c}\text { Não } \\
\text { suspeitos }\end{array}$ & \\
\hline $1^{\circ}$ período & $9(81,8)$ & $2(18,2)$ & & $12(54,5)$ & $10(45,5)$ & \\
\hline $2^{\circ}$ período & $3(60,0)$ & $2(40,0)$ & & $16(84,2)$ & $3(15,8)$ & \\
\hline $3^{\circ}$ período & $7(100,0)$ & $0(0,0)$ & 0,65 & $6(75,0)$ & $2(25,0)$ & 0,67 \\
\hline $4^{\circ}$ período & $4(57,1)$ & $3(42,9)$ & & $19(100,0)$ & $0(0,0)$ & \\
\hline $5^{\circ}$ período & $4(80,0)$ & $1(20,0)$ & & $13(65,0)$ & $7(35,0)$ & \\
\hline p-valor ${ }^{1}$ & \multicolumn{2}{|c|}{0,04} & \multicolumn{4}{|c|}{0,04} \\
\hline
\end{tabular}

Valores em $n(\%) . p$-valor ${ }^{1}=$ diferença entre suspeitos e não suspeitos; $p$-valor ${ }^{2}=$ diferença entre as séries do curso.

Ao analisar a presença de transtornos mentais comuns nos alunos de acordo com o tipo de metodologia, foi possível notar que não houve diferença entre os casos suspeitos de acordo com o SQR-20, pois em ambas as metodologias utilizadas o número de casos suspeitos é grande (Tabela 4).

Tabela 4. Análise comparativa do SQR-20 dos alunos do curso de Fisioterapia da UEPA, Belém, Pará, Brasil.

\begin{tabular}{lccc}
\hline \multirow{2}{*}{ Tipo de metodologia } & \multicolumn{2}{c}{ SRQ-20 } & \multirow{2}{*}{ Total } \\
\cline { 2 - 3 } & Suspeitos & Não suspeitos & \\
\hline MA & $53(73,6)$ & $19(26,4)$ & $72(100)$ \\
MT & $40(78,4)$ & $11(21,6)$ & $51(100)$ \\
p-valor & \multicolumn{3}{c}{0,68} \\
Valores em n (\%). MA - Metodologia ativa; MT - metodologia tradicional.
\end{tabular}

Por fim, ao se buscar a relação do SRQ-20 com a prática de atividade extracurricular, lazer e atividade física dos alunos foi encontrado resultados interessantes. Quando se 
analisou estratificando por sexo e por período do curso, não foi encontrado nenhuma relação significante, assim como quando estratificado apenas pelo tipo de metodologia. Porém, ao se analisar estratificando por metodologia e por sexo, as alunas pertencentes à MA e que declaram não praticar atividade física aumenta em 4,80 vezes a chance de transtorno mental, fato este não observado no grupo MT. E quando se analisou todos os alunos juntos, foi possível notar que aqueles alunos que não praticam atividade física aumentam em 2,85 vezes a chance de transtorno mental (Tabela 5).

\section{DISCUSSÃO}

A investigação dos transtornos mentais entre estudantes é um assunto relevante. Por meio desta pesquisa foi possível encontrar informações importantes acerca deste assunto, ou seja, as variáveis sociodemográficas não denotaram maior propensão a estes distúrbios, diferente de outros estudos ${ }^{4,5}$.

No que se trata da faixa etária deste estudo, o público foi considerado como adolescentes e adultos jovens, os quais já são mais propensos ao desenvolvimento de estresse. Tal motivo está relacionado ao contato com diversos estressores comumente encontrados nesta fase da vida ${ }^{3}$. Ademais, nessa fase é comum que haja uma intensificação dos processos de maturação psicológica do indivíduo diante do meio social ${ }^{8}$. 
Tabela 5. Relação dos fatores de risco com os transtornos mentais comuns nos alunos do curso de Fisioterapia da UEPA.

\begin{tabular}{|c|c|c|c|c|c|c|}
\hline \multirow{2}{*}{ Variáveis } & \multicolumn{2}{|c|}{$\begin{array}{c}\text { Atividade } \\
\text { Extracurricular }\end{array}$} & \multicolumn{2}{|c|}{ Lazer } & \multicolumn{2}{|c|}{$\begin{array}{c}\text { Atividade } \\
\text { Física }\end{array}$} \\
\hline & Sim & Não & Sim & Não & Sim & Não \\
\hline \multicolumn{7}{|l|}{ MA(Masculino) } \\
\hline Suspeito & 16 & 3 & 16 & 3 & 14 & 5 \\
\hline Não suspeito & 3 & 1 & 4 & 0 & 4 & 0 \\
\hline OR (IC95\%) & \multicolumn{2}{|c|}{$0,56(-)$} & \multicolumn{2}{|c|}{ SE } & \multicolumn{2}{|c|}{ SE } \\
\hline p-valor & \multicolumn{2}{|c|}{0,77} & \multicolumn{2}{|c|}{ SE } & \multicolumn{2}{|c|}{ SE } \\
\hline \multicolumn{7}{|l|}{ MA (Feminino) } \\
\hline Suspeito & 31 & 3 & 19 & 15 & 10 & 24 \\
\hline Não suspeito & 14 & 1 & 11 & 4 & 10 & 5 \\
\hline OR (IC95\%) & \multicolumn{2}{|c|}{$1,35(0,12-14,20)$} & \multicolumn{2}{|c|}{$2,17(0,57-8,20)$} & \multicolumn{2}{|c|}{$4,80(1,30-17,65)$} \\
\hline p-valor & \multicolumn{2}{|c|}{0,75} & \multicolumn{2}{|c|}{0,40} & \multicolumn{2}{|c|}{0,03} \\
\hline \multicolumn{7}{|l|}{ MT (Masculino) } \\
\hline Suspeito & 9 & 1 & 8 & 2 & 8 & 2 \\
\hline Não suspeito & 2 & 0 & 0 & 2 & 2 & 0 \\
\hline OR (IC95\%) & \multicolumn{2}{|c|}{ SE } & \multicolumn{2}{|c|}{ SE } & \multicolumn{2}{|c|}{ SE } \\
\hline p-valor & \multicolumn{2}{|c|}{ SE } & \multicolumn{2}{|c|}{ SE } & \multicolumn{2}{|c|}{ SE } \\
\hline \multicolumn{7}{|l|}{ MT (Feminino) } \\
\hline Suspeito & 28 & 3 & 15 & 16 & 13 & 18 \\
\hline Não suspeito & 6 & 2 & 6 & 2 & 5 & 3 \\
\hline OR (IC95\%) & \multicolumn{2}{|c|}{$0,32(0,04-2,36)$} & \multicolumn{2}{|c|}{$3,20(0,55-18,38)$} & \multicolumn{2}{|c|}{$2,30(0,46-11,42)$} \\
\hline p-valor & \multicolumn{2}{|c|}{0,57} & \multicolumn{2}{|c|}{0,34} & \multicolumn{2}{|c|}{0,52} \\
\hline Alunos do cursc & & & & & & \\
\hline Suspeito & 84 & 10 & 58 & 36 & 45 & 49 \\
\hline Não suspeito & 25 & 4 & 21 & 8 & 21 & 8 \\
\hline OR (IC95\%) & 0,74 & $-2,57)$ & 1,62 & $5-4,06)$ & 2,85 & $5-7,09)$ \\
\hline p-valor & & & & & & \\
\hline
\end{tabular}

MA - Metodologia ativa; MT - Metodologia tradicional. SE - Não foi possível fazer estatística. (-) sem intervalo de confiança. (OR) Razão de Chances. (IC95\%) Intervalo de confiança a 95\%.

Em relação ao sexo o público feminino é o mais presente, fato que pode propiciar a prevalência de transtornos mentais nesse público, principalmente relacionadas ao estresse emocional ${ }^{9}$. Outro estudo também mostra que a sobrecarga das atividades acadêmicas se torna um fator de diminuição do tempo para outras atividades, fato 
este que pode influenciar na não participação em atividades de lazer, como nos achados deste estudo ${ }^{10}$.

A sobrecarga acadêmica pode ser observada na existência de fatores de risco à saúde mental de alunos desde o período inicial até períodos intermediários da formação, seja esta baseada no uso de metodologias ativas, seja pelo uso da metodologia tradicional. De modo que esta cobrança perpassa de suas respectivas grades curriculares, visto que um grande contingente de estudantes se insere no exercício de atividades extracurriculares ${ }^{11}$.

Nos casos de sobrecarga durante os períodos intermediários da formação, deve-se levar em consideração que muitos universitários exerçam alguma atividade laboral ou estejam em busca de uma, visando ter um suporte e até mesmo sua própria subsistência, tendo em vista suas condições financeiras. Ademais, a sobrecarga acadêmica como um todo, afeta não somente o lazer dos estudantes, mas também a prática de atividade física. Sabe-se que a atividade física tem sido amplamente debatida e relacionada à promoção de saúde, tendo repercussões na prevenção de diversas disfunções físicas e mentais, como o estresse e ansiedade. Tal fator pode ser observado neste estudo com a análise de todos os alunos, sendo que os que não realizam atividade física apresentam um aumento em 2,85 vezes a chance de transtorno mental. Logo, é perceptível que tal diminuição da prática de atividade física pode repercutir negativamente na Qualidade de Vida da população universitária ${ }^{3}$. 
Apesar da metodologia ativa representar um desafio maior para alguns alunos, devido ainda estar sendo implementada nas universidades e divergir do estilo de ensino perpassado pelos indivíduos por um longo período, incluindo o ensino fundamental e médio, não houve diferença entre os casos suspeitos de transtorno mental entre as metodologias. Tal resultado permite colocar o tipo de metodologia de ensino como um fator secundário aos possíveis transtornos mentais nessa pesquisa. É possível que por exigir maior interação, ela seja um problema para pessoas mais tímidas, porém também é provável que seja benéfica às mesmas pessoas a longo prazo, com 0 desenvolvimento pessoal relacionado principalmente à comunicação e proatividade ${ }^{12}$.

Já o sistema avaliativo em si, encontrado em ambas as metodologias expostas, acaba sendo um grande obstáculo, que por sua vez gera medo, angústia, estresse e ansiedade na maioria dos alunos. Parte desses sentimentos e condições estão relacionados a cobranças internas e externas e podem afetar ainda o humor e autoestima dos universitários ${ }^{3}$.

Além dos fatores discutidos, a própria inserção em uma universidade torna-se um período de mudanças e desafios, as insatisfações pessoais, cobranças internas e externas podem ser fatores estressantes. Dessa forma, o apoio emocional, atividades de lazer e práticas de atividade física que minorem tais fatores seriam de suma importância para que os estudantes perpassassem por tal ocasião de forma mais positiva. Além de um suporte advindo das instâncias de 
ensino, diminuindo a evasão e a presença de transtornos mentais, viabilizando uma formação integral e melhor QV aos discentes.

Não se pode negar que é importante que novas pesquisas sejam realizadas a fim de investigar de fato a existência de algum problema mental específico, para que se possa traçar estratégias que ajudem o aluno. Aponta-se ainda que há limitações no estudo por utilizar escalas subjetivas principalmente relacionadas a uma temática relevante, mas pode adquirir informações relevantes para o rastreamento destes transtornos e encaminhar para serviços especializados.

\section{CONCLUSÃO}

Pode-se concluir que os alunos que não praticam atividade física aumentam em 2,85 vezes a chance de desenvolver transtorno mental. E quando estratificado por sexo e por metodologia, foi observado que as alunas pertencentes a metodologia ativa e que não praticam atividade física, aumentam em 4,8 vezes a chance de desenvolver transtornos mentais.

\section{REFERÊNCIAS}

1. Hiany N, Vieira MA, Gusmão ROM, Barbosa SFA. Perfil epidemiológico dos transtornos mentais na população adulta no Brasil: uma revisão integrativa. Rev Enferm Atual 2018;86. https://doi.org/10.31011/reaid-2018-v.86-n.24-art.676

2. Oliveira LA, Oliveira VC. Os transtornos depressivos: um olhar sobre a reestruturação dos cuidados em saúde mental. Rev Psicol Saúde Debate 2018;4:96-109. https://doi.org/10.22289/2446-922X.V4N3A9 
3. Oliveira LS. Qualidade de vida e saúde mental de estudantes universitários (Dissertação). Ceará: Universidade Federal do Ceará; 2020, $145 \mathrm{p}$.

http://www.repositorio.ufc.br/bitstream/riufc/53459/3/2020 dis Isoli veira.pdf

4.Graner KM, Cerqueira ATAR. Revisão integrativa: sofrimento psíquico em estudantes universitários e fatores associados. Cienc Saúde Col 2019;24:1327-46. https://doi.org/10.1590/1413-

81232018244.09692017

5.Araújo FM, Machado MP. Ansiedade, estresse e depressão associado ao índice de rendimento acadêmico em estudantes de fisioterapia (monografia). Juiz de fora: Universidade Federal de Juiz de Fora; 2018, 39 p. https://www.ufjf.br/facfisio/files/2019/03/ansiedade-estresse-edepress \%c3\%83o-associado-ao-\%c3\%8dndice-de-rendimento-

academico-em-estudantes-de-fisioterapia.pdf

6.Gonçalves DM, Stein AT, Kapczinski F. Avaliação de desempenho do Self-Reporting Questionnaire como instrumento de rastreamento psiquiátrico: um estudo comparativo com o Structured Clinical Interview for DSM-IV-TR. Cad Saúde Pub 2008;24:38090. https://doi.org/10.1590/S0102-311X2008000200017

7. Harding TW, Arango MV, Baltazar J, Climent CE, Ibrahim HH, Ladrido-Ignacio L, et al. Mental disorders in primary health care: a study of their frequency and diagnosis in four developing countries. Psychol Med 1980;10:231-41.

https://doi.org/10.1017/s0033291700043993

8. Kebede MA, Anbessie B, Ayano G. Prevalence and predictors of depressivo and axiety among medical students in Addis Av Ana, Ethiopia. Int M J Health Syst 2019;13:30. https://doi.org/10.1186/s13033-019-0287-6

9.Saldaña OC, Loera SLA, Madrigal TBE. Evaluation of Stress Academic Levels of Medical Students of The South University Center. Case: Ciudad Guzman. Cienc Trab 2017;19:31-4.

http://dx.doi.org/10.4067/S0718-24492017000100031

10.Lora GP, Golin CS, Lise AMR, Linartevichi VF. Avaliação da saúde mental de graduandos de medicina de uma instituição particular de ensino superior do oeste do estado do Paraná. FAG J Heath 2020;3:357-63. https://doi.org/10.35984/fjh.v2i3.231

11.Souza M, Caldas T, De Antoni C. Fatores de adoecimento dos estudantes da área da saúde: uma revisão sistemática. Psicodebate 2017;3:99-126.

http://psicodebate.dpgpsifpm.com.br/index.php/periodico/article/vie w/93

12. Mattar J, Aguiar APS. Metodologias Ativas: aprendizagem baseada em problemas, problematização e método do caso. Bras J Ed Tech Soc 2018;11:404-15. http://dx.doi.org/10.14571/brajets.v11.n3.404-415 